\title{
On nonsingularity of circulant matrices
}

\author{
Zhangchi Chen
}

December 9, 2020

\begin{abstract}
In Communication theory and Coding, it is expected that certain circulant matrices having $k$ ones and $k+1$ zeros in the first row are nonsingular. We prove that such matrices are always nonsingular when $2 k+1$ is either a power of a prime, or a product of two distinct primes. For any other integer $2 k+1$ we construct circulant matrices having determinant 0 . The smallest singular matrix appears when $2 k+1=45$. The possibility for such matrices to be singular is rather low, smaller than $10^{-4}$ in this case.

Keywords: Circulant matrices, Cyclotomic polynomials, Communication theory, Coding

MSC classifications: 15B05, 11R18, 68P30, 94A05
\end{abstract}

\section{Introduction}

We begin with definitions and some classical results on circulant matrices, taken from ([2] Section 1).

Definition 1.1. A circulant matrix $C\left(a_{0}, \ldots, a_{n-1}\right)$ is an $n \times n$ matrix of the form

$$
\left[\begin{array}{cccc}
a_{0} & a_{1} & \ldots & a_{n-1} \\
a_{n-1} & a_{0} & \ldots & a_{n-2} \\
\vdots & \vdots & \ddots & \vdots \\
a_{1} & a_{2} & \ldots & a_{0}
\end{array}\right]
$$

where $a_{0}, \ldots, a_{n-1} \in \mathbb{Q}$. It is unital if $a_{0}, \ldots, a_{n-1} \in\{0,1\}$.

The determinant of a circulant matrix $C\left(a_{0}, \ldots, a_{n-1}\right)$ can be calculated by

$$
\operatorname{det} C\left(a_{0}, \ldots, a_{n-1}\right)=\prod_{j=0}^{n-1}\left(a_{0}+a_{1} \omega_{j}+a_{2} \omega_{j}^{2}+\ldots a_{n-1} \omega_{j}^{n-1}\right),
$$

in terms of the $n$-th root of unity

$$
\omega_{j}:=e^{\frac{2 \pi i j}{n}}, \quad\left(i^{2}=-1\right) .
$$

A proof can be found in ([1] Theorem 4.8.2). 
Definition 1.2. The polynomial

$$
f(x)=a_{0}+a_{1} x+a_{2} x^{2}+\cdots+a_{n-1} x^{n-1} \in \mathbb{Q}[x]
$$

is called the associated polynomial of $C\left(a_{0}, \ldots, a_{n-1}\right)$. It is called unital if all its coefficients $a_{0}, \ldots, a_{n-1}$ are in $\{0,1\}$.

Note that the relation between circulant matrices and their associated polynomials is not ' $1-1$ '. For example the identity matrix of any size has $f(x)=1$. In fact, a circulant matrix is determined by both its associated polynomial and its size.

Proposition 1.3. A circulant matrix $C\left(a_{0}, \ldots, a_{n-1}\right)$ is nonsingular if and only if its associated polynomial $f(x)$ and $x^{n}-1$ share no common roots.

Proof. By what precedes:

$$
\operatorname{det} C\left(a_{0}, \ldots, a_{n-1}\right)=\prod_{j=0}^{n-1} f\left(\omega_{j}\right) .
$$

Definition 1.4. A circulant matrix $C\left(a_{0}, \ldots, a_{n-1}\right)$ is called $r$-recurrent with $r$ a proper divisor of $n$, if $a_{j}=a_{j^{\prime}}$ whenever $j \equiv j^{\prime} \bmod r$. It is called non-recurrent if it is not $r$-recurrent for any proper divisor $r$ of $n$.

If $C\left(a_{0}, \ldots, a_{n-1}\right)$ is $r$-recurrent for some proper divisor $r$ of $n$, then it has the same rank as $C\left(a_{0}, \ldots, a_{r-1}\right)$ because

$$
C\left(a_{0}, \ldots, a_{n-1}\right)=\left[\begin{array}{cccc}
C\left(a_{0}, \ldots, a_{r-1}\right) & C\left(a_{0}, \ldots, a_{r-1}\right) & \ldots & C\left(a_{0}, \ldots, a_{r-1}\right) \\
C\left(a_{0}, \ldots, a_{r-1}\right) & C\left(a_{0}, \ldots, a_{r-1}\right) & \ldots & C\left(a_{0}, \ldots, a_{r-1}\right) \\
\vdots & \vdots & \ddots & \vdots \\
C\left(a_{0}, \ldots, a_{r-1}\right) & C\left(a_{0}, \ldots, a_{r-1}\right) & \ldots & C\left(a_{0}, \ldots, a_{r-1}\right)
\end{array}\right] .
$$

Hence an invertible circulant matrix must be non-recurrent. This paper mainly studies circulant unital matrices having $k$ ones and $k+1$ zeros in the first row. They are always non-recurrent.

One motivation to study such matrices comes from Communication and Coding where $2 k+1$ input signals are 'mixed' by these matrices [5, Theorem 13, Remark 5], 6, Theorem 29]. Experts hope such processes to be invertible. Another motivation comes from [4] on summability of polydiagonal matrices for periodic sequences of zeros and ones. The following two questions are equivalent:

Question 1.5. For fixed $k \in \mathbb{Z}_{\geqslant 1}$, does there exist a circulant unital matrix having $k$ ones and $k+1$ zeros in its first row which is singular?

Question 1.6. For fixed $k \in \mathbb{Z}_{\geqslant 1}$, does there exist a unital polynomial $f(x) \in \mathbb{Z}[x]$ such that $\operatorname{deg} f(x) \leqslant 2 k, f(1)=k$ and $f(x)$ shares a common root with $x^{n}-1$ ?

In this paper we propose a complete answer.

Theorem 1.7. If $2 k+1=p^{e}$ for some prime $p$, then such matrices are always nonsingular.

Theorem 1.8. If $2 k+1=p q$ for two distinct primes $3 \leqslant p<q$, then such matrices are always nonsingular.

Theorem 1.9. If $2 k+1=p q r$ where $3 \leqslant p<q$ are two distinct primes and $r \geqslant 3$ is an odd integer, then there exist some singular matrices of such a type. 
Corollary 1.10. The first singular example appears when $(p, q, r)=(3,5,3)$, i.e. $2 k+1=45$. For example if

$$
\begin{aligned}
E_{22}:= & \{0,9,18,27,36, \\
& 3,12,21,30,39, \\
& 1,16,31, \\
& 2,17,32, \\
& 4,19,34, \\
& 5,20,35\} .
\end{aligned}
$$

and if $a_{j}=1$ for $j \in E_{22}$ and $a_{j}=0$ for $j \notin E_{22}$, then $C\left(a_{0}, \ldots, a_{44}\right)$ is singular.

Proof. Let $f_{E_{22}}(x) \in \mathbb{Z}[x]$ be the associated polynomial of this matrix. Then

$$
\begin{aligned}
f_{E_{22}}(x)=\sum_{j \in E_{22}} x^{j} & =\left(1+x^{3}\right)\left(1+x^{9}+x^{18}+x^{27}+x^{36}\right)+\left(x+x^{2}+x^{4}+x^{5}\right)\left(1+x^{15}+x^{30}\right) \\
& =\left(1+x^{3}\right) \frac{x^{45}-1}{x^{9}-1}+\left(x+x^{2}+x^{4}+x^{5}\right) \frac{x^{45}-1}{x^{15}-1}
\end{aligned}
$$

annihilates $e^{\frac{2 \pi i}{45}}$, a root of $x^{45}-1$.

\section{Cyclotomic polynomials}

By Proposition 1.3, $C\left(a_{0}, \ldots, a_{n-1}\right)$ is singular if and only if $f(x)$ and $x^{n}-1$ have a common root. Thus we should study the irreducible and the unital factors of $x^{n}-1$, namely the cyclotomic polynomials and the fundamental recurrent polynomials (Definition 4.1).

Definition 2.1. For any $n \in \mathbb{Z}_{\geqslant 1}$, the $n$-th cyclotomic polynomial is defined as

$$
\Phi_{n}(x):=\prod_{\substack{1 \leqslant d \leqslant n \\ \operatorname{gcd}(d, n)=1}}\left(x-e^{\frac{2 \pi i d}{n}}\right) \in \mathbb{C}[x] .
$$

We recall the following properties ([3, VI. 3]).

- (Degree) $\operatorname{deg} \Phi_{n}(x)=\varphi(n)$ where $\varphi$ is Euler's totient function.

- (Integer coefficients) $\Phi_{n}(x) \in \mathbb{Z}[x]$.

- (Irreducibility) $\Phi_{n}(x)$ is irreducible over $\mathbb{Z}[x]$.

- (Factorization) $x^{n}-1=\prod_{r \mid n} \Phi_{r}(x)$.

- For any two distinct $n, m \in \mathbb{Z}_{\geqslant 1}, \Phi_{n}(x)$ and $\Phi_{m}(x)$ do not divide one another.

- For a prime number $p$ and for $e \in \mathbb{Z}_{\geqslant 1}$, we have $\Phi_{p^{e}}(x)=1+x^{p^{e-1}}+x^{2 p^{e-1}}+\cdots+x^{p^{e}-p^{e-1}}$. Hence $\Phi_{p^{e}}(1)=p$. Note that $x^{p^{e}}-1=\Phi_{1}(x) \Phi_{p}(x) \ldots \Phi_{p^{e}}(x)$.

Theorem 2.2. A circulant matrix of size $n \times n$ is singular if and only if its associated polynomial $f(x)$ is divisible by $\Phi_{d}(x)$ for some $d$ dividing $n$. 


\section{Case where $2 k+1$ is a power of a prime}

From ([3, IV. 2, Corollary 2.2]), recall the Gauss Lemma: if a monic polynomial $f(x) \in \mathbb{Z}[x]$ is equal to $h(x) g(x)$ with $g(x) \in \mathbb{Z}[x]$ monic and $h(x) \in \mathbb{Q}[x]$, then $h(x) \in \mathbb{Z}[x]$ has integer coefficients.

Lemma 3.1. Let $f(x)$ be a unital polynomial, and let $p$ be a prime number not dividing $f(1)$. Then $\Phi_{p^{e}}(x)$ does not divide $f(x)$ for any $e \in \mathbb{Z}_{\geqslant 0}$.

Proof. When $e=0$, since $f(1) \in \mathbb{Z}_{\geqslant 1}$ is nonzero, $\Phi_{1}(x)=x-1$ cannot divide $f(x)$. When $e \in$ $\mathbb{Z}_{\geqslant 1}$, suppose $g(x)=\Phi_{p^{e}}(x)$ divides $f(x)$ for some $e \in \mathbb{Z}_{\geqslant 1}$, so that by the Gauss Lemma, $f(x)=$ $h(x) g(x)$ with $h(x) \in \mathbb{Z}[x]$. Hence $f(1)=h(1) g(1)=h(1) p$, contradicting the hypothesis that $p$ does not divide $f(1)$.

Proof of Theorem 1.7. Write $2 k+1=p^{e}$ with $p$ an odd prime and $e \in \mathbb{Z}_{\geqslant 1}$. Let $f(x) \in$ $\mathbb{Z}[x]$ be a unital polynomial with $\operatorname{deg} f(x)<p^{e}-1$ and $f(1)=k$. Remember $x^{p^{e}}-1=$ $\Phi_{1}(x) \Phi_{p}(x) \ldots \Phi_{p^{e}}(x)$. Since $p$ does not divide $k$, by Lemma 3.1, $\Phi_{p^{e^{\prime}}}(x)$ does not divide $f(x)$ for any $e^{\prime} \in \mathbb{Z}_{\geqslant 0}$. Thus $f(x)$ shares no common root with $x^{p^{e}}-1$, i.e. the corresponding circulant matrix is nonsingular.

\section{Recurrent decompositions}

Next we introduce the unital factors of $x^{n}-1$ : the fundamental recurrent polynomials. The following notation is taken from Ingleton [2].

Definition 4.1. For any proper divisor $r$ of $n$, the fundamental $r$-recurrent polynomial with respect to $n$ is

$$
G(n, r ; x):=\frac{x^{n}-1}{x^{r}-1}=1+x^{r}+\cdots+x^{n-r} \in \mathbb{Z}[x] .
$$

The name comes from the following fact: for any $r$-recurrent $n \times n$ circulant matrix, the associated polynomial $f(x)$ is a multiple of $G(n, r ; x)$.

We have

$$
G(n, r ; x)=\prod_{d \mid n} \Phi_{d}(x) / \prod_{d \mid r} \Phi_{d}(x)=\prod_{\substack{d \mid n \\ d \nmid r}} \Phi_{d}(x),
$$

and in particular, $\Phi_{n}(x)$ divides $G(n, r ; x)$.

For example $G(45,9 ; x)=\Phi_{45}(x) \Phi_{15}(x) \Phi_{5}(x)$.

In the previous section, note that we have decomposed

$$
f_{E_{22}}(x)=\left(1+x^{3}\right) G(45,9 ; x)+\left(x+x^{2}+x^{4}+x^{5}\right) G(45,15 ; x) .
$$

In general, if $f(x) \in \mathbb{Q}[x]$ with $\operatorname{deg} f(x)<n$ can be decomposed as $\sum_{\substack{r \mid n \\ r<n}} h_{r}(x) G(n, r ; x)$ for some $h_{r}(x) \in \mathbb{C}[x]$, then clearly $\Phi_{n}(x)$ divides $f(x)$.

In this section we want to establish the converse. For any $f(x) \in \mathbb{Q}[x]$ with $\operatorname{deg} f(x)<n$, which is divisible by $\Phi_{n}(x)$, we want to decompose it as $\sum_{\substack{r \mid n \\ r<n}} h_{r}(x) G(n, r ; x)$. 
Definition 4.2. (Recurrent decomposition) Fix $n \in \mathbb{Z}_{\geqslant 1}$ and let $f(x) \in \mathbb{Q}[x]$ with $\operatorname{deg} f(x)<n$. Let $p_{1}<\cdots<p_{m}$ be all the distinct prime factors of $n$. If

$$
f(x)=\sum_{j=1}^{m} h_{\frac{n}{p_{j}}}(x) G\left(n, \frac{n}{p_{j}} ; x\right),
$$

for some $h_{\frac{n}{p_{j}}}(x) \in \mathbb{Q}[x]$ with $\operatorname{deg} h_{\frac{n}{p_{j}}}(x)<\frac{n}{p_{j}}$, then we call $\left(h_{\frac{n}{p_{1}}}(x), \ldots, h_{\frac{n}{p_{m}}}(x)\right) a\left(p_{1}, \ldots, p_{m}\right)$ recurrent decomposition of $f(x)$ with respect to $n$. We call such a decomposition unital if each $h_{\frac{n}{p_{j}}}(x)$ is unital.

For example $\left(x+x^{2}+x^{4}+x^{5}, 1+x^{3}\right)$ is a unital $(3,5)$-recurrent decomposition of $f_{E_{22}}(x)$ with respect to 45 .

The following Theorem is equivalent to [2, Proposition 3.1].

Theorem 4.3. (Existence of a recurrent decomposition) Let $f(x) \in \mathbb{Q}[x]$ with $\operatorname{deg} f(x)<n$. Suppose $\Phi_{n}(x)$ divides $f(x)$. Let $p_{1}<\cdots<p_{m}$ be all the distinct prime factors of $n$. Then $f(x)$ admits a $\left(p_{1}, \ldots, p_{m}\right)$-recurrent decomposition with respect to $n$.

Proof. Using (11), we have

$$
\operatorname{gcd}\left(\left\{G\left(n, \frac{n}{p_{j}} ; x\right): j=1, \ldots, m\right\}\right)=\operatorname{gcd}\left(\left\{\prod_{d \mid n, d \nmid \frac{n}{p_{j}}} \Phi_{d}(x): j=1, \ldots, m\right\}\right)=\Phi_{n}(x) .
$$

Applying the Euclidean Algorithm we find some $g_{\frac{n}{p_{j}}}(x) \in \mathbb{Q}[x]$ such that

$$
\Phi_{n}(x)=\sum_{j=1}^{m} g_{\frac{n}{p_{j}}}(x) G\left(n, \frac{n}{p_{j}} ; x\right) .
$$

Suppose $f(x)=q(x) \Phi_{n}(x)$ where $q(x) \in \mathbb{Q}[x]$. Then $f(x)=\sum_{j=1}^{m} q(x) g_{\frac{n}{p_{j}}}(x) G\left(n, \frac{n}{p_{j}} ; x\right)$. We now need to change the multiplicators $q(x) g_{\frac{n}{p_{j}}}(x)$ to meet the degree bounds.

The quotient

$$
\frac{x^{n}-1}{G\left(n, \frac{n}{p_{j}} ; x\right)}=x^{\frac{n}{p_{j}}}-1
$$

is a polynomial of degree $\frac{n}{p_{j}}$. By Euclidean division, there exists a unique $h_{\frac{n}{p_{j}}}(x) \in \mathbb{Q}[x]$ with $\operatorname{deg} h_{\frac{n}{p_{j}}}(x)<\frac{n}{p_{j}}$ such that $q(x) g_{\frac{n}{p_{j}}}(x) \equiv h_{\frac{n}{p_{j}}}(x) \bmod x^{\frac{n}{p_{j}}}-1$. Then

$$
f(x)=\sum_{j=1}^{m} q(x) g_{\frac{n}{p_{j}}}(x) G\left(n, \frac{n}{p_{j}} ; x\right) \equiv \sum_{j=1}^{m} h_{\frac{n}{p_{j}}}(x) G\left(n, \frac{n}{p_{j}} ; x\right) \bmod x^{n}-1 .
$$

Since $\operatorname{deg} f(x)<n$ we conclude that $f(x)=\sum_{j=1}^{m} h_{\frac{n}{p_{j}}}(x) G\left(n, \frac{n}{p_{j}} ; x\right)$.

When $f(x)$ is unital, we expect the existence of a unital decomposition. This is not true in general. A counterexample where $m=3$ will be constructed at the end of the section. The case where $m=2$ is proved by Ingleton [2, 4.1]. We prove a stronger version here.

We write $\mathbb{Z}_{[0, d]}[x]$ for the set of polynomials having coefficients in $\{0,1, \ldots, d\}$. 
Theorem 4.4. (Recurrent decomposition in $\mathbb{Z}_{[0, d]}[x]$ ) Let $p<q$ be two distinct primes. Let $n=p^{e_{1}} q^{e_{2}}$ with $e_{1}, e_{2} \in \mathbb{Z}_{\geqslant 1}$. Let $f(x) \in \mathbb{Z}_{[0, d]}[x]$ with $\operatorname{deg} f(x)<n$ be a polynomial which is divisible by $\Phi_{n}(x)$. Then $f(x)$ admits a $(p, q)$-recurrent decomposition in $\mathbb{Z}_{[0, d]}[x]$ with respect to $n$.

In particular, when $d=1$, a unital decomposition exists.

Proof. By Theorem 4.3, there exist $h_{\frac{n}{p}}(x), h_{\frac{n}{q}}(x) \in \mathbb{Q}[x]$ with $\operatorname{deg} h_{\frac{n}{p}}(x)<\frac{n}{p}$, $\operatorname{deg} h_{\frac{n}{q}}(x)<\frac{n}{q}$ such that $f(x)=h_{\frac{n}{p}}(x) G\left(n, \frac{n}{p} ; x\right)+h_{\frac{n}{q}}(x) G\left(n, \frac{n}{q} ; x\right)$. Write

$$
\begin{aligned}
& f(x)= \sum_{j=0}^{n-1} a_{j} x^{j}=\sum_{s=0}^{\frac{n}{p q}-1} x^{s}\left(\sum_{l=0}^{p q-1} a_{\frac{l n}{p q}+s} x^{\frac{l n}{p q}}\right), \\
& h_{\frac{n}{p}}(x)=\sum_{u=0}^{\frac{n}{p}-1} b_{u} x^{u}=\sum_{s=0}^{\frac{n}{p q}-1} x^{s}\left(\sum_{l=0}^{q-1} b_{\frac{l n}{p q}+s} x^{\frac{l n}{p q}}\right), \\
& h_{\frac{n}{q}}(x)=\sum_{v=0}^{\frac{n}{q}-1} c_{v} x^{v}=\sum_{s=0}^{\frac{n}{p q}-1} x^{s}\left(\sum_{l=0}^{p-1} c_{\frac{l n}{p q}+s} x^{\frac{l n}{p q}}\right) .
\end{aligned}
$$

For every $s=0, \ldots, \frac{n}{p q}-1$, we group the coefficients as

$$
\begin{aligned}
& A_{s}:=\left\{a_{\frac{l n}{p q}+s}: l=0, \ldots, p q-1\right\} \subset\{0, \ldots, d\}, \\
& B_{s}:=\left\{b_{\frac{l n}{p q}+s}: l=0, \ldots, q-1\right\} \subset \mathbb{Q}, \\
& C_{s}:=\left\{c_{\frac{l n}{p q}+s}: l=0, \ldots, p-1\right\} \subset \mathbb{Q} .
\end{aligned}
$$

Define $e_{s}:=\min B_{s} \in \mathbb{Q}$ and introduce

$$
g(x):=\sum_{s=0}^{\frac{n}{p q}-1} e_{s} x^{s} .
$$

Using

$$
G\left(\frac{n}{p}, \frac{n}{p q} ; x\right)=\sum_{l=0}^{q-1} x^{\frac{l n}{p q}}, \quad G\left(\frac{n}{q}, \frac{n}{p q} ; x\right)=\sum_{l=0}^{p-1} x^{\frac{l n}{p q}},
$$

we may subtract and modify the multiplicators as

$$
\begin{aligned}
& h_{\frac{n}{p}}^{\prime}(x):=h_{\frac{n}{p}}(x)-g(x) G\left(\frac{n}{p}, \frac{n}{p q} ; x\right)=\sum_{s=0}^{\frac{n}{p q}-1} x^{s}\left(\sum_{l=0}^{q-1}\left(b_{\frac{l n}{p q}+s}-e_{s}\right) x^{\frac{l n}{p q}}\right), \\
& h_{\frac{n}{q}}^{\prime}(x):=h_{\frac{n}{q}}(x)+g(x) G\left(\frac{n}{q}, \frac{n}{p q} ; x\right)=\sum_{s=0}^{\frac{n}{p q}-1} x^{s}\left(\sum_{l=0}^{p-1}\left(c_{\frac{l n}{p q}+s}+e_{s}\right) x^{\frac{l n}{p q}}\right) .
\end{aligned}
$$

Since $G\left(\frac{n}{p}, \frac{n}{p q} ; x\right) G\left(n, \frac{n}{p} ; x\right)=G\left(\frac{n}{q}, \frac{n}{p q} ; x\right) G\left(n, \frac{n}{q} ; x\right)$, we see that $\left(h_{\frac{n}{p}}^{\prime}(x), h_{\frac{n}{q}}^{\prime}(x)\right)$ is another decomposition of $f(x)$. So we can suppose from the beginning that $\min B_{s}=0$ for all $s$.

Given two sets of rational numbers $X, Y \subset \mathbb{Q}$, define their sum and difference by

$$
X+Y:=\{x+y: x \in X, y \in Y\}, \quad X-Y:=\{x-y: x \in X, y \in Y\} .
$$


We have $X \subset(X+Y)-Y$. From

$$
\begin{aligned}
& h_{\frac{n}{p}}(x) G\left(n, \frac{n}{p} ; x\right)=\sum_{s=0}^{\frac{n}{p q}-1} x^{s}\left(\sum_{l=0}^{q-1} b_{\frac{l n}{p q}+s}\left(x^{\frac{l n}{p q}}+x^{\frac{(l+q) n}{p q}}+\cdots+x^{\frac{(l+(p-1) q) n}{p q}}\right)\right), \\
& h_{\frac{n}{q}}(x) G\left(n, \frac{n}{q} ; x\right)=\sum_{s=0}^{\frac{n}{p q}-1} x^{s}\left(\sum_{l=0}^{p-1} c_{\frac{l n}{p q}+s}\left(x^{\frac{l n}{p q}}+x^{\frac{(l+p) n}{p q}}+\cdots+x^{\frac{(l+(q-1) p) n}{p q}}\right)\right),
\end{aligned}
$$

we deduce that each coefficient $a_{\frac{l_{1} n}{p q}+s} \in A_{s}, l_{1} \in\{0, \ldots, p q-1\}$ is a sum of the form

$$
a_{\frac{l_{1} n}{p q}+s}=b_{\frac{l_{2} n}{p q}+s}+c_{\frac{l_{3} n}{p q}+s}
$$

where $l_{2} \in\{0, \ldots, q-1\}, l_{3} \in\{0, \ldots, p-1\}$ such that $l_{1} \equiv l_{2} \bmod q$ and $l_{1} \equiv l_{3} \bmod p$. Thus by the Chinese Reminder Theorem,

$$
B_{s}+C_{s}=A_{s} \subset\{0,1, \ldots, d\} .
$$

Since $\min B_{s}=0$ we have $C_{s}=\{0\}+C_{s} \subset B_{s}+C_{s} \subset\{0,1, \ldots, d\}$ and $B_{s} \subset\left(A_{s}-C_{s}\right) \cap \mathbb{Q}_{\geqslant 0}=$ $\{-d,-d+1, \ldots, d\} \cap \mathbb{Q}_{\geqslant 0}=\{0,1, \ldots, d\}$, i.e. both $h_{\frac{n}{p}}(x)$ and $h_{\frac{n}{q}}(x)$ are in $\mathbb{Z}_{[0, d]}[x]$.

Example 4.5. If $n$ has more than two distinct prime factors, Theorem 4.4 may be false, i.e. a unital polynomial $f(x)$ divisible by $\Phi_{n}(x)$ may not admit any unital recurrent decomposition. A counterexample appears when $n=105=3 \times 5 \times 7$ and

$$
\begin{aligned}
f(x) & =x^{5}+x^{6}+x^{10}+x^{25}+x^{27}+x^{35}+x^{40}+x^{48}+x^{50}+x^{65}+x^{69}+x^{70}+x^{80}+x^{85}+x^{95}+x^{100} \\
& =\left(1+x^{5}+x^{10}+x^{15}+x^{25}+x^{30}\right) G(105,35 ; x)+x^{6} G(105,21 ; x)-G(105,15 ; x) .
\end{aligned}
$$

Here $f(x)$ is unital and $f(1)=16$. However if we suppose $f(x)=h_{35}(x) G(105,35 ; x)+$ $h_{21}(x) G(105,21 ; x)+h_{15}(x) G(105,15 ; x)$ for some unital $h_{35}(x), h_{21}(x)$ and $h_{15}(x)$, then $16=$ $3 h_{35}(1)+5 h_{21}(1)+7 h_{15}(1)$, where $h_{35}(1), h_{21}(1), h_{15}(1) \in \mathbb{Z}_{\geqslant 0}$. The only solutions $\left(h_{35}(1), h_{21}(1), h_{15}(1)\right)$ to this equation are $(0,2,2)$ and $(1,0,3)$, hence either $h_{21}(1)$ or $h_{15}(1)$ is 0 , i.e. either $h_{21}$ or $h_{15}$ is 0 since they are unital. In the first case $e^{\frac{2 \pi i}{15}}$ is a root of $h_{35}(x) G(105,35 ; x)+h_{21}(x) G(105,21 ; x)$ but not a root of $f(x)$. In the second case $e^{\frac{2 \pi i}{21}}$ is a root of $h_{35}(x) G(105,35 ; x)+h_{15}(x) G(105,15 ; x)$ but not a root of $f(x)$. These contradictions prove that $f(x)$ admits no unital recurrent decomposition.

Table 1: The values of each polynomial at 3 special points

\begin{tabular}{l|c|c|c|c}
\hline & $G(105,35 ; x)$ & $G(105,21 ; x)$ & $G(105,15 ; x)$ & $f(x)$ \\
\hline$x=e^{\frac{2 \pi \imath}{35}}$ & 3 & 0 & 0 & $-3 e^{\frac{8 \pi \imath}{7}}$ \\
\hline$x=e^{\frac{2 \pi \imath}{21}}$ & 0 & 5 & 0 & $5 e^{\frac{6 \pi \tau}{7}}$ \\
\hline$x=e^{\frac{2 \pi \imath}{15}}$ & 0 & 0 & 7 & -7 \\
\hline
\end{tabular}




\section{Case where $2 k+1$ is a product of two distinct primes}

Proof of Theorem 1.8. Write $2 k+1=p q$, where $p, q$ are two distinct primes. Suppose $C\left(a_{0}, \ldots, a_{2 k}\right)$ is singular. Let $f(x) \in \mathbb{Z}_{[0,1]}[x]$ be its corresponding unital polynomial. Since neither $p$ nor $q$ divides $k$, by Lemma 3.1 we know none among $\Phi_{1}(x), \Phi_{p}(x)$ and $\Phi_{q}(x)$ divides $f(x)$. So $\Phi_{p q}(x)$ divides $f(x)$. By Theorem 4.4 we have

$$
f(x)=h_{q}(x) G(p q, q ; x)+h_{p}(x) G(p q, p ; x),
$$

for some $h_{q}(x), h_{p}(x)$ unital. As before, we write

$$
f(x)=\sum_{j=0}^{p q-1} a_{j} x^{j}, \quad h_{q}(x)=\sum_{u=0}^{q-1} b_{u} x^{u}, \quad h_{p}(x)=\sum_{v=0}^{p-1} c_{v} x^{v},
$$

and write

$$
\begin{aligned}
& A_{0}:=\left\{a_{l}: l=0, \ldots, p q-1\right\}, \\
& B_{0}:=\left\{b_{l}: l=0, \ldots, q-1\right\}, \\
& C_{0}:=\left\{c_{l}: l=0, \ldots, p-1\right\} .
\end{aligned}
$$

We have $k=f(1)=h_{q}(1) q+h_{p}(1) p$. Since neither $p$ nor $q$ divides $k$, we know $h_{q}(1) \neq 0$, $h_{p}(1) \neq 0$, hence $1 \in B_{0}, 1 \in C_{0}$. By the Chinese Reminder Theorem we know $B_{0}+C_{0}=A_{0}$, hence $2 \in A_{0}$, contradicting the hypothesis that $f(x)$ is unital.

\section{Other cases: constructing a singular matrix}

Proof of Theorem 1.9. In all other cases we can write $2 k+1=p q r$ where $p, q$ are two distinct primes and $r \geqslant 3$ is an odd integer. We may assume $p<q$ and $p \leqslant r$ by choosing $p, q$ as the first and the second smallest prime factors of $2 k+1$. To construct a singular circulant matrix of our type, it suffices to find a unital polynomial $f(x) \in \mathbb{Z}[x]$ with $\operatorname{deg} f(x)<p q r, f(1)=k$ such that $\Phi_{p q r}(x)$ divides $f(x)$. Since $k=\frac{p q r-1}{2} \geqslant \frac{3 p q-1}{2}>p q$, we have unique $a, b \in \mathbb{Z}_{\geqslant 1}$ with $b \leqslant p-1$ such that $a p+b q=k$. Define

$$
f(x):=\left(1+x^{q r}+x^{2 q r}+\cdots+x^{b q r-q r}\right) G(p q r, p r)(x)+\sum_{j \in R_{a}} x^{j} G(p q r, q r)(x),
$$

where $R_{a} \subset\{0,1, \ldots, q r-1\} \backslash\{0, r, \ldots, q r-r\}$ is a set of $a$ elements. Such $R_{a}$ exists if and only if $a \leqslant q r-q$. This is true since

$$
\begin{aligned}
q r-q-a & =q r-q-\frac{k-b q}{p} \\
& =q r-q-\frac{p q r-1-b q}{2 p} \\
& =\frac{q r}{2}-q+\frac{1+b q}{2 p} \\
& \geqslant \frac{r-2}{2} q>0 .
\end{aligned}
$$

Clearly we have $f(x) \in \mathbb{Z}[x]$ and $\operatorname{deg} f(x)<p q r$. Since $\Phi_{p q r}(x)$ divides both $G(p q r, p r ; x)$ and $G(p q r, q r ; x)$ it also divides $f(x)$. Moreover $f(1)=b G(p q r, p r)(1)+a G(p q r, q r)(1)=$ $b q+a p=k$. The condition $R_{a} \cap\{0, r, \ldots, q r-r\}=\emptyset$ ensures that $f(x)$ is unital. 
The smallest $k$ in this case is 22 , when $2 k+1=45=3^{2} \times 5$. Here we take $p=r=3, q=5$. Note that $22=4 \times 3+2 \times 5$. In this case we can construct a singular matrix as above, by taking $R_{4}=\{1,2,4,5\}$. Then $f(x)=\left(1+x^{3}\right)\left(1+x^{9}+x^{18}+x^{27}+x^{36}\right)+\left(x+x^{2}+x^{4}+x^{5}\right)\left(1+x^{15}+x^{30}\right)$ represents a $45 \times 45$ singular circulant unital matrix with 22 ones in its first row. This reveals how we constructed $E_{22}$ in Corollary 1.10 .

\section{The number of singular matrices when $k=22$}

Finally we count the number of such singular matrices and estimate the probability of a circulant matrix of our type to be singular. We study the easiest case when $k=22$. There are in total $\left(\begin{array}{l}45 \\ 22\end{array}\right)=4116715363800$ choices of such matrices. Suppose $f(x)$ is a corresponding polynomial of a singular circulant matrix of our type. Then there exists some $r$ dividing 45 such that $\Phi_{r}(x)$ divides $f(x)$. Since neither 3 nor 5 divides $f(1)$, by Lemma 3.1 we know none among $\Phi_{1}(x), \Phi_{3}(x), \Phi_{5}(x)$ and $\Phi_{9}(x)$ divides $f(x)$. Thus either $\Phi_{15}(x)$ or $\Phi_{45}(x)$ divides $f(x)$.

\subsection{Case (1): $\Phi_{45}(x)$ divides $f(x)$}

Theorem 4.4 guarantees the existence of unital recurrent decompositions of $f(x)$, but not the uniqueness. However, we can always control the ambiguity.

Theorem 7.1. Suppose $n=p^{e_{1}} q^{e_{2}}$ has two distinct prime factors $p, q$. If $f(x) \in \mathbb{Q}[x]$ with $\operatorname{deg} f(x)$ divisible by $\Phi_{n}(x)$, then for any two $(p, q)$-recurrent decompositions $\left(h_{\frac{n}{p}}(x), h_{\frac{n}{q}}(x)\right)$ and $\left(h_{\frac{n}{p}}^{\prime}(x), h_{\frac{n}{q}}^{\prime}(x)\right)$ of $f(x)$ with respect to $n$ such that

$$
\operatorname{deg} h_{\frac{n}{p}}(x)<\frac{n}{p}, \quad \operatorname{deg} h_{\frac{n}{p}}^{\prime}(x)<\frac{n}{p}, \quad \operatorname{deg} h_{\frac{n}{q}}(x)<\frac{n}{q}, \quad \operatorname{deg} h_{\frac{n}{q}}^{\prime}(x)<\frac{n}{q},
$$

there exists some $\delta(x) \in \mathbb{Q}[x]$ with $\operatorname{deg} \delta(x)<\frac{n}{p q}$ such that

$$
h_{\frac{n}{p}}^{\prime}(x)=h_{\frac{n}{p}}(x)-\delta(x) G\left(\frac{n}{p}, \frac{n}{p q} ; x\right), \quad h_{\frac{n}{q}}^{\prime}(x)=h_{\frac{n}{q}}(x)+\delta(x) G\left(\frac{n}{q}, \frac{n}{p q} ; x\right) .
$$

Proof. By the definition of $(p, q)$-recurrent decompositions with respect to $n$, we have

$$
\left(h_{\frac{n}{p}}(x)-h_{\frac{n}{p}}^{\prime}(x)\right) G\left(n, \frac{n}{p} ; x\right)=\left(h_{\frac{n}{q}}^{\prime}(x)-h_{\frac{n}{q}}(x)\right) G\left(n, \frac{n}{q} ; x\right) .
$$

Note that

$$
\begin{aligned}
& G\left(n, \frac{n}{p} ; x\right)=\Phi_{n}(x) \prod_{j=1}^{e_{2}} \Phi_{n q^{-j}}(x)=\Phi_{n}(x) G\left(\frac{n}{q}, \frac{n}{p q} ; x\right), \\
& G\left(n, \frac{n}{q} ; x\right)=\Phi_{n}(x) \prod_{j=1}^{e_{1}} \Phi_{n p^{-j}}(x)=\Phi_{n}(x) G\left(\frac{n}{p}, \frac{n}{p q} ; x\right) .
\end{aligned}
$$

Hence $G\left(\frac{n}{q}, \frac{n}{p q} ; x\right)$ and $G\left(\frac{n}{p}, \frac{n}{p q} ; x\right)$ share no nontrivial common factors. Dividing both sides of (41) by $\Phi_{n}(x)$, we get

$$
\left(h_{\frac{n}{p}}(x)-h_{\frac{n}{p}}^{\prime}(x)\right) G\left(\frac{n}{q}, \frac{n}{p q} ; x\right)=\left(h_{\frac{n}{q}}^{\prime}(x)-h_{\frac{n}{q}}(x)\right) G\left(\frac{n}{p}, \frac{n}{p q} ; x\right) .
$$

So there exists some $\delta(x) \in \mathbb{Q}[x]$ such that

$$
h_{\frac{n}{p}}(x)-h_{\frac{n}{p}}^{\prime}(x)=\delta(x) G\left(\frac{n}{p}, \frac{n}{p q} ; x\right) \quad h_{\frac{n}{q}}^{\prime}(x)-h_{\frac{n}{q}}(x)=\delta(x) G\left(\frac{n}{q}, \frac{n}{p q} ; x\right) .
$$

We have $\operatorname{deg} \delta(x) \leqslant \max \left\{h_{\frac{n}{p}}(x), h_{\frac{n}{p}}^{\prime}(x)\right\}-\operatorname{deg} G\left(\frac{n}{p}, \frac{n}{p q} ; x\right)<\frac{n}{p}-\left(\frac{n}{p}-\frac{n}{p q}\right)=\frac{n}{p q}$. 
Definition 7.2. As in the proof of Theorem4.4, for any $(p, q)$-recurrent decomposition $\left(h_{\frac{n}{p}}(x), h_{\frac{n}{q}}(x)\right)$ of $f(x)$ with respect to $n$, write

$$
h_{\frac{n}{p}}(x)=\sum_{u=0}^{\frac{n}{p}-1} b_{u} x^{u},
$$

and for any $s=0, \ldots, \frac{n}{p q}-1$ define

$$
B_{s}:=\left\{b_{\frac{l n}{p q}+s}: l=0, \ldots, q-1\right\} .
$$

We call a $(p, q)$-recurrent decomposition $p$-uniformized if $\min B_{s}=0$ for any $s=0, \ldots, \frac{n}{p q}-1$.

Corollary 7.3. Suppose $n=p^{e_{1}} q^{e_{2}}$ has two distinct prime factors $p, q$. Let $f(x) \in \mathbb{Q}[x]$ with $\operatorname{deg} f(x)<n$ be divisible by $\Phi_{n}(x)$. Then among all the $(p, q)$-recurrent decompositions of $f(x)$ with respect to $n$, there exists a unique p-uniformized one. Moreover, if $f(x)$ is unital, its p-uniformized decomposition is also unital.

Proof. (Existence) For any $(p, q)$-recurrent decomposition $\left(h_{\frac{n}{p}}(x), h_{\frac{n}{q}}(x)\right)$ of $f(x)$ with respect to $n$, define $e_{s}:=\min B_{s} \in \mathbb{Q}, g(x):=\sum_{s=0}^{\frac{n}{p q}-1} e_{s} x^{s}$. Then $\left(h \frac{n}{p}(x)-g(x) G\left(\frac{n}{p}, \frac{n}{p q} ; x\right), h_{\frac{n}{q}}(x)+\right.$ $\left.g(x) G\left(\frac{n}{q}, \frac{n}{p q} ; x\right)\right)$ is $p$-uniformized.

(Uniqueness) If there are two $p$-uniformized $(p, q)$-recurrent decompositions $\left(h_{\frac{n}{p}}(x), h_{\frac{n}{q}}(x)\right)$ and $\left(h_{\frac{n}{p}}^{\prime}(x), h_{\frac{n}{q}}^{\prime}(x)\right)$ of $f(x)$ with respect to $n$, by Theorem 7.1 there exists some $\delta(x) \in \mathbb{Q}[x]$ with $\operatorname{deg} \delta(x)<\frac{n}{p q}$ satisfying (3).

Write $B_{s}, B_{s}^{\prime}$ as a collection of coefficients of $h_{\frac{n}{p}}^{\prime}(x)$ and $h_{\frac{n}{p}}^{\prime}(x)$ respectively, as in Definition 7.2. Write $\delta(x)=\sum_{s=0}^{\frac{n}{p q}-1} \delta_{s} x^{s}$. From (3) we have $\min B_{s}^{\prime}=\min B_{s}-\delta_{s}$. So $\delta_{s}=0$ for all $s=0, \ldots, \frac{n}{p q}-1$. We conclude that these two decompositions are equivalent.

(Unital) In Theorem 4.4 we have already constructed a $p$-uniformized unital $(p, q)$-recurrent decomposition when $f(x)$ is unital.

By Corollary 7.3 we have a unique 3-uniformized unital $(3,5)$-recurrent decomposition of $f(x)$ with respect to 45 , i.e.

$$
f(x)=h_{15}(x) G(45,15 ; x)+h_{9}(x) G(45,9 ; x),
$$

where $h_{15}(x), h_{9}(x) \in \mathbb{Z}[x]$ are unital and if we write

$$
f(x)=\sum_{j=0}^{44} a_{j} x^{j}, \quad h_{15}(x)=\sum_{u=0}^{14} b_{u} x^{u}, \quad h_{9}(x)=\sum_{v=0}^{8} c_{v} x^{v},
$$

for $s=0,1,2$ we may define

$$
\begin{aligned}
& A_{s}:=\left\{a_{3 l+s}: l=0, \ldots, 14\right\} \subset\{0,1\}, \\
& B_{s}:=\left\{b_{3 l+s}: l=0, \ldots, 4\right\} \subset\{0,1\}, \\
& C_{s}:=\left\{c_{3 l+s}: l=0, \ldots, 2\right\} \subset\{0,1\} .
\end{aligned}
$$

Here we have $\min B_{s}=0$ for any $s=0,1,2$. Moreover $22=f(1)=3 h_{15}(1)+5 h_{9}(1)$ where $h_{15}(1), h_{9}(1) \in \mathbb{Z}_{\geqslant 0}$, hence $h_{15}(1)=4, h_{9}(1)=2$.

Each $f(x)$ corresponds to a unique choice of $b_{0}, \ldots, b_{14}, c_{0}, \ldots, c_{8} \in\{0,1\}$ satisfying the following conditions: 
- $\sum_{u=0}^{14} b_{u}=4, \sum_{v=0}^{8} c_{v}=2$,

- $\min B_{s}=0$,

- if $1 \in B_{s}$ then $C_{s}=\{0\}$.

Case (1.1): there is only $1 s$ such that $1 \in C_{s}$. There are $\left(\begin{array}{l}3 \\ 1\end{array}\right)\left(\begin{array}{l}3 \\ 2\end{array}\right)\left(\begin{array}{c}10 \\ 4\end{array}\right)=1890$ choices.

Case (1.2): there are $2 s$ such that $1 \in C_{s}$. There are $\left(\begin{array}{l}3 \\ 2\end{array}\right)\left(\begin{array}{l}3 \\ 1\end{array}\right)^{2}\left(\begin{array}{l}5 \\ 4\end{array}\right)=135$ choices.

Hence there are 2025 choices of unital $f(x)$ divisible by $\Phi_{45}(x)$, corresponding to 2025 singular matrices.

\subsection{Case (2): $\Phi_{15}(x)$ divides $f(x)$}

Write $f^{(15)}(x):=\sum_{j=0}^{14} d_{j} x^{j} \in \mathbb{Z}[x]$ where $d_{j}:=a_{j}+a_{j+15}+a_{j+30} \in\{0,1,2,3\}$. In fact it is the residue of $f(x)$ divided by $x^{15}-1$, hence divisible by $\Phi_{15}(x)$. Such $f^{(15)}(x)$ corresponds to $\prod_{j=0}^{14}\left(\begin{array}{l}3 \\ d_{j}\end{array}\right)$ unital $f(x)$. For any $f^{(15)}(x)$ divisible by $\Phi_{15}(x)$, by Corollary 7.3 it admits a unique 3 -uniformized unital $(3,5)$-recurrent decomposition with respect to 15 :

$$
f^{(15)}(x)=h_{5}(x) G(15,5 ; x)+h_{3}(x) G(15,3 ; x),
$$

where $h_{5}(x), h_{3}(x) \in \mathbb{Q}[x]$ and if we write

$$
h_{5}(x)=\sum_{u=0}^{4} b_{u} x^{u}, \quad h_{3}(x)=\sum_{v=0}^{2} c_{v} x^{v},
$$

define

$$
\begin{aligned}
& A_{0}:=\left\{d_{l}: l=0, \ldots, 14\right\} \subset\{0,1,2,3\}, \\
& B_{0}:=\left\{b_{l}: l=0, \ldots, 4\right\}, \\
& C_{0}:=\left\{c_{l}: l=0, \ldots, 2\right\} .
\end{aligned}
$$

Then $\min B_{0}=0$. By the Chinese Reminder Theorem we have $B_{0}+C_{0}=A_{0}$. Thus $C_{0}=\{0\}+$ $C_{0} \subset B_{0}+C_{0} \subset\{0,1,2,3\}$ and $B_{0} \subset[0,+\infty) \cap\left(A_{0}+\{0,-1,-2,-3\}\right)=\{0,1,2,3\}$. Moreover we have $f^{(15)}(1)=22=3 h_{5}(1)+5 h_{3}(1)$ where $h_{3}(1), h_{5}(1) \in \mathbb{Z}_{\geqslant 0}$, hence $h_{5}(1)=4, h_{3}(1)=2$.

Each $f^{(15)}(x)$ corresponds to a unique choice of $b_{0}, \ldots, b_{4}, c_{0}, \ldots, c_{2} \in\{0,1,2,3\}$ satisfying the following conditions:

1. $\sum_{u=0}^{4} b_{u}=4, \sum_{v=0}^{2} c_{v}=2$,

2. $\min B_{0}=0$,

3. $\max B_{0}+\max C_{0} \leqslant 3$.

We can write down a complete list of possible values of $\left(b_{u}\right),\left(c_{v}\right)$ up to permutations. Note that

- each $f^{(15)}(x)$ is uniquely determined by values of $\left(b_{u}\right),\left(c_{v}\right)$;

- for each $f^{(15)}(x)=\sum_{j=0}^{14} d_{j} x^{j}$ there are $\prod_{j=0}^{14}\left(\begin{array}{l}3 \\ d_{j}\end{array}\right)=3^{\#\left\{j: d_{j}=1,2\right\}}$ choices of unital $f(x)$.

By summing up (Permutations) $\times\left(\right.$ Choices of $f(x)$ for each $\left.f^{(15)}(x)\right)$ we get 88376670 choices of unital $f(x)$ divisible by $\Phi_{15}(x)$, corresponding to 88376670 singular matrices. 
Table 2: Possible values of $\left(b_{0}, \ldots, b_{4}\right),\left(c_{0}, c_{1}, c_{2}\right)$ up to permutations

\begin{tabular}{c|c|c}
\hline Type of values & Permutations & Choices of $f(x)$ for each $f^{(15)}(x)$ \\
\hline$(0,0,0,2,2),(0,1,1)$ & $\left(\begin{array}{l}5 \\
2\end{array}\right)\left(\begin{array}{l}3 \\
2\end{array}\right)=60$ & $3^{6} \times 3^{2}=3^{8}$ \\
\hline$(0,0,1,1,2),(0,1,1)$ & $\left(\begin{array}{c}5 \\
1\end{array}\right)\left(\begin{array}{l}4 \\
2\end{array}\right)\left(\begin{array}{l}3 \\
2\end{array}\right)=90$ & $3^{6} \times 3^{5}=3^{11}$ \\
\hline$(0,1,1,1,1),(0,0,2)$ & $\left(\begin{array}{l}5 \\
4\end{array}\right)\left(\begin{array}{l}3 \\
1\end{array}\right)=15$ & $3^{8} \times 3^{1}=3^{9}$ \\
\hline$(0,1,1,1,1),(0,1,1)$ & $\left(\begin{array}{l}5 \\
4\end{array}\right)\left(\begin{array}{l}3 \\
2\end{array}\right)=15$ & $3^{6} \times 3^{8}=3^{14}$ \\
\hline
\end{tabular}

\subsection{Double counts: both $\Phi_{15}(x)$ and $\Phi_{45}(x)$ divide $f(x)$}

Suppose a unital $f(x)$ with $\operatorname{deg} f(x)<45$ is divisible by both $\Phi_{15}(x)$ and $\Phi_{45}(x)$. As in case (1) there exist some unital $h_{15}(x), h_{9}(x)$ such that

$$
f(x)=h_{15}(x) G(45,15 ; x)+h_{9}(x) G(45,9 ; x),
$$

with $h_{15}(1)=4$. Take value at $\zeta_{15}:=e^{\frac{2 \pi i}{15}}$. Since $f(x)$ is divisible by $\Phi_{15}(x)$ we have $f\left(\zeta_{15}\right)=0$. We also have $G\left(45,15 ; \zeta_{15}\right)=3, G\left(45,9 ; \zeta_{15}\right)=0$. Hence $h_{15}\left(\zeta_{15}\right)=0$. We conclude that $h_{15}(x)$ is divisible by $\Phi_{15}(x)$. It is also unital and $\operatorname{deg} h_{15}(x)<15$. By Theorem 4.4, $h_{15}(x)$ admits a unital $(3,5)$-recurrent decomposition with respect to 15 :

$$
h_{15}(x)=h_{5}(x) G(15,5 ; x)+h_{3}(x) G(15,3 ; x),
$$

for some unital $h_{5}(x), h_{3}(x) \in \mathbb{Z}[x]$ with $\operatorname{deg} h_{5}(x)<5$, deg $h_{3}(x)<3$.

We have $4=h_{15}(1)=3 h_{5}(1)+5 h_{3}(1)$, where $h_{5}(1), h_{3}(1) \in \mathbb{Z}_{\geqslant 0}$. However there is no solution $\left(h_{5}(1), h_{3}(1)\right)$ to this equation. We conclude that there is no unital $f(x)$ with $\operatorname{deg} f(x)<$ $45, f(1)=22$ and $f(x)$ divisible by both $\Phi_{15}(x)$ and $\Phi_{45}(x)$.

We have 88378695 singular unital circulant matrices having exactly 22 ones in their first rows. The possibility of a unital circulant matrix in our type being singular is about $2.15 \times 10^{-5}<10^{-4}$. This algorithm can be generalized to all $n$ with only 2 distinct prime factors.

\section{References}

[1] Golub, G. H.; Van Loan, C. F.: Matrix computations, Fourth edition. Johns Hopkins Studies in the Mathematical Sciences. Johns Hopkins University Press, Baltimore, MD, (2013). xiv+756 pp.

[2] Ingleton, A. W.: The rank of circulant matrices, J. London Math. Soc. 31 (1956), 632-635.

[3] Lang, S.: Algebra, Revised third edition. Graduate Texts in Mathematics, 211. Springer-Verlag, New York, (2002). $\mathrm{xvi}+914 \mathrm{pp}$.

[4] Newton, R. H. C.: On the summation of periodic sequences. I, II, Nederl. Akad. Wetensch. Proc. Ser. A. 57 = Indag. Math. 16, (1954). 533-544, 545-549.

[5] Wan, K; Tuninetti D; Ji M; Piantanida P.: Combination Networks with End-user-caches: Novel Achievable and Converse Bounds under Uncoded Cache Placement, arxiv.org/abs/1701.06884/, 49 pages.

[6] Wan, K: Limites fondamentales de stockage pour les réseaux de diffusion de liens partagés et les réseaux de combinaison, theses.fr/2018SACLS217/, 146 pages. 